\title{
Recent Advances in Elongated and Round Spermatid Injection
}

\author{
(D) Fatih Gökalp, (1) Sadık Görür \\ Hatay Mustafa Kemal University, Faculty of Medicine, Department of Urology, Hatay, Turkiye
}

\section{What's known on the subject? and What does the study add?}

Azoospermia is a condition that is characterized by absence of spermatozoa in the ejaculate, occurs in 15\% of infertile men. Classified as obstructive azoospermia and non-obstructive azoospermia. Prior to microdissection testicular sperm extraction (microTESE) and in vitro fertilization/microinjection defined, donor insemination was the only option for non-obstructive azoospermia patients. However, the success rate of microTESE is reported up to $60 \%$ for all cases and this results forced the clinicians consider another possibilities such as the injection of early spermatids; elongated spermatids and round spermatids into oocytes. Although most of the studies are animal experiments, it has been shown that round spermatid administration can also cause fertility in humans. However, the round spermatid injection had lower success rate compared to elongated spermatid injection. This review demonstrated that the success rates of round spermatid injections are not as high as elongated spermatid injections. The he most critical factor affecting the success is correct cell selection and proper transfer. Thus, round spermatid injection success rates can approach elongated spermatid injection when carried out absolutely correctly. Although congenital anomalies are rarely reported after spermatid injection, the risk is known to be higher than in natural conception.

\section{Abstract}

Azoospermia is commonly identified in patients with infertility. Non-obstructive azoospermia (NOA) includes primary or secondary and incomplete testicular failure. Before testicular sperm extraction (TESE), donor insemination is the only option available in men with NOA. The combination of microTESE and intracytoplasmic sperm injection has considerably increased the fertilization rate. However, mature spermatozoa can be found in half of the patients. This situation prompted experts to use spermatids to assist in reproductive techniques. Elongated spermatid injection (ELSI) and round spermatid injection (ROSI) are among the possible treatments for couples who cannot find mature spermatozoa after microTESE in patients with NOA. This review provides an updated summary of the most recent available topics on ELSI and ROSI in the literature.

Keywords: Infertility, spermatogenesis, spermatids, testicular function

\section{Introduction}

Infertility is a significant health problem affecting 20\% of couples. Azoospermia is seen in $\sim 15 \%$ of infertile men and is divided into two classes, obstructive azoospermia (OA) and non-obstructive azoospermia (NOA) (1). Patients with OA have normal spermatogenesis in the testis; however, spermatozoa are absent in the semen due to an obstruction in any part of the genital system. Patients with NOA have insufficient or hesitant spermatogenesis. Spermatogenesis is a complex process starting from mitosis of the spermatogonia to haploid round spermatid meiosis. Spermatogenesis then undergoes condensation and elongation in the spermiogenesis stage, where the head and tail of the sperm cell are formed (2). These structural changes are critical stages for a living sperm cell. Should there be pauses in these stages, mature spermatozoa do not form.

NOA is often due to primary testicular insufficiency [increased follicle-stimulating hormone (FSH) and luteinizing hormone (LH) levels accompanying small testicles], secondary testicular insufficiency (low FSH and LH levels associated with small testicles due to hypogonadotropic hypogonadism), or incomplete or ambiguous genitals (increased FSH levels or small testicular size accompanying average FSH levels) (1). Before the availability of microdissection testicular sperm extraction (microTESE) and intracytoplasmic sperm injection (ICSI), the only recommended option for patients who did not respond to medical treatment was donor insemination. Gratifyingly, because of the combination of microTESE and ICSI accompanying

Correspondence: Fatih Gökalp MD, Hatay Mustafa Kemal University, Faculty of Medicine, Department of Urology, Hatay, Turkiye

Phone: + 903262291000 E-mail: fatihgokalp85@gmail.com 0RCID-ID: orcid.org/0000-0003-3099-3317

Received: 03.02.2021

Accepted: 11.04.2021

Cite this article as: Gökalp F, Görür S. Recent Advances in Elongated and Round Spermatid Injection. J Urol Surg 2021;8(4):234-237.

${ }^{\circ}$ Copyright 2021 by the Association of Urological Surgery / Journal of Urological Surgery published by Galenos Publishing House. 
laboratory and genetic tests, the possibility to obtain sperm has increased considerably (3). However, spermatozoa are found in only approximately half of the cases after microTESE, even in experienced centers; therefore, the transfer of cells before maturation was tried in spermiogenesis to achieve fertilization in patients without spermatozoa, and evidence has shown that fertilization was achieved with elongated spermatid (ELS) and round spermatids (ROS) $(4,5)$.

\section{Spermiogenesis}

Spermiogenesis is the final stage of spermatogenesis, where haploid ROS turns into mature motile spermatozoa (6). Spermatids contain Golgi apparatus, mitochondria, centrioles, and a nucleus. These formations are important in the shape of mature spermatozoa. The function of the Golgi apparatus plays an especially crucial role in spermiogenesis (7). Spermiogenesis can be divided into four basic stages: 1) the Golgi phase; 2) acrosome formation; 3) tail formation; and 4) maturation. Acrosome formation, which provides the egg's penetration into the protein sheath, is an early and essential stage performed by the Golgi apparatus. While vesicles within the cell unite to form the acrosome, the radially symmetrical spermatids become polarized. The Golgi apparatus then creates enzymes that will make up the acrosome. Later, the confluent acrosome vesicle begins to grow on the nuclear membrane surface and covers half of the membrane surface. The Golgi apparatus takes a cap by covering the core that passes to the center's other side. Once the acrosome is formed, a centriole of the sperm cell elongates to form the tail. This tail becomes a modified and mobile cilium (8). As spermiogenesis continues, the core is compressed and elongated. Cuff formation develops in the distal part with core densification and elongation. In the last stage of maturation, Sertoli cells phagocytose excess cytoplasm.

\section{Cell Selection for ELSI and ROSI}

The probability of finding viable spermatozoa after microTESE varies between $40 \%$ and $60 \%$ in patients with NOA; however, no mature spermatozoa can be found in half of these patients. This situation prompted experts to use spermatids to assist in reproductive techniques, resulting in a breakthrough in infertility treatments. Research showed that pregnancy could be achieved after the injection of spermatids into oocytes $(4,9)$.

Fertilization success rates vary after elongated spermatid injection (ELSI) and round spermatid injection (ROSI), which can be obtained due to abnormal progression in spermiogenesis or maturation arrest. Early studies used the Papanicolaou test, fluorescence labeling, Pisum sativum agglutinin binding, and antiacrosin antiserum immune labeling for cell selection; however, living cells could not be obtained by employing these separation methods (10). Then, protocols, such as the Percoll gradient centrifuge, which enables the separation based on cell density, the STA-PUT velocity sedimentation based on miRNA fluorescence-assisted cell sorting (FACS) based on DNA content, or propidium iodide, a DNA intercalation dye, were implemented for the effective and specific isolation of pure germ cells at different developmental stages (11-14). Differentiating them using the FACS method is difficult because the cell DNA content is similar. Instead, the selection of germ cells according to cell density is more efficient.

Currently, the most successful method for cell selection employs discernment under an electron microscope. Cells are selected based on their fundamental structural differences. Since ELS cells have a different head structure, they can be identified more easily than ROS cells. ROS are the smallest spermatogenic cells with a dimension of nearly 6-8 $\mu \mathrm{m}$. ROS cells do not have distinct nucleoli, and the edge of the cytoplasm surrounding the nucleus is thinner than in spermatogonium. Active pseudopods seen in spermatogonia are also absent in round spermatocytes. Additionally, the cytoplasm of ROS is easily separated from the nucleus when pulled back and forth in the pipette. The fluorescence in situ hybridization (FISH) method is also helpful in identifying ROS cells. Mendoza et al. (15) used immunochemical imaging of proacrosis and autosomal DNA FISH to identify ROS. All ROS spermatids with pro-acrosine activity were haploid in the FISH. When the authors expanded their research, they emphasized that cell size is the main criterion for ROS selection. Computer-aided identification of live spermatids is predicted to be available soon.

\section{ELSI and ROSI Success Rates}

ELSI and ROSI success rates in patients with NOA are essential factors in transferring these cells. The literature showed that fertilization can be achieved with ROSI; however, early studies demonstrated the low efficiency of this process $(10,16)$. The popularity of these procedures has decreased over time, especially since the American Reproductive Medicine Practice Committee defines ROSI as an experimental study (17). Similar fertilization rates were reported in ROSI and ELSI. However, these rates are lower than the fertilization rates obtained with mature spermatozoa obtained from microTESE $(16,17)$. Data showed that fertilization rates after the transfer of the ELS cells with better maturation are slightly better than ROSI (18). A retrospective study by Sousa et al. (19) revealed that fertilization rates were $71.4 \%, 53.6 \%$, and $17 \%$ in patients with ICSI, ELSI, and ROSI, respectively, and clinical pregnancy rates were $31.7 \%, 26.3 \%$, and $0 \%$, respectively. Additionally, in the same study, when the literature was reviewed, fertilization rates after ELSI and ROSI were $48.4 \%$ and $21.8 \%$, and pregnancy rates were $28.9 \%$ and $2.8 \%$, respectively. In seminal work by Tanaka et al. (5), the fertilization rate after ROSI was applied to 86 female patients was $76.4 \%$, with a total pregnancy rate of $16.2 \%$ in cells whose spermatids were cryopreserved before transfer. In their later study, they showed that the fertilization 
rate was $60.2 \%$ in those who were cryopreserved and with a low total number of pregnancies (9.4\%) (17).

The most important factor affecting the success is correct cell selection $(5,17,20)$. In animal models, ROS were defined as cells formed that contain central chromatin. Yet, human ROS do not contain central chromatin, and this situation supports that early studies' failure was the wrong cell selection. ELS can be selected more easily based on their DNA material and structural features; however, this selection is difficult for ROS. The morphological structure of ROS has been defined. According to the changes it has undergone, the terms early-ROS and late-ROS are used (21).

Another important factor affecting the success is the incomplete activation of oocytes due to insufficient activation ability of ROS; therefore, Tanaka et al. $(5,17)$ reported that success rates increased when they activated oocytes after transfer. Another factor affecting the success was when the transfer was made, whether fresh spermatid or after freezing. The literature shows that transfer made after freezing the spermatids increased fertilization success since the uterine endometrium can be better prepared and the transfer can occur when it is in the best condition (5).

\section{Safety of ELSI and ROSI}

The reliability of assisted reproductive techniques depends on another important step. Spermatids may be susceptible to genetic disorders, which may occur in late gametogenesis and contribute to embryonic development (22). An early study showed that DNA methylation, essential for genomic imprinting, was not completed at the ROS stage; however, DNA methylation can still be completed after injection. This theory is supported by the observation of DNA methylation fluctuations that were completed during early embryonic development. DNA methylation and demethylation occur throughout spermatogenesis and mostly before meiosis I (23). Some researchers thought that the failure of ROSI was due to a lack of DNA methylation in ROS. Still, in animal models, DNA methylation is complete in ROS $(23,24)$. In a study by Bonduelle et al. (25), which included 2889 pediatric patients with ICSI and 2995 with IVF, malformation rates were 1.69\% and 1.31\%, respectively. A review by Ludwig and Diedrich (26) reported that the rate of major malformation increased by $8.6 \%$ and the relative risk increased by $1.25 \%$ in the ICSI cases. In Tanaka et al. (17) study, which included 90 infants from ROSI with a 2-year follow-up, congenital anomalies were found in 3 (3.3\%) of them (1 cleft palate, 1 ventricular septal defect, and 1 omphalocele). The authors stated that they did not evaluate whether there was a statistical difference between the standard delivery groups during this period due to the small number of ROSI groups. However, ROSI babies did have a low birth weight. At the end of the 2 years, no significant difference was found between the groups in terms of syndrome (Prader-Willi syndrome, Angelman syndrome, Wiskott-Alrich syndrome, etc.) or abnormal physical or mental developmental disorders. Nevertheless, healthcare providers should explain parents in planning the pregnancy before the spermatid transfer the risks of possible hereditary diseases, such as Prader-Willi and Angelman syndromes $(17,22)$.

\section{Conclusion}

ELSI and ROSI are among the possible treatments for couples who cannot find mature spermatozoa after micro-TESE in patients with NOA. The success rates of ROSI are not as high as ELSI; however, the most critical factor affecting the success is correct cell selection and proper transfer. Thus, ROSI success rates can approach ELSI when performed correctly. Congenital anomalies are rarely reported after spermatid injection; however, the risk is higher than in natural conception. With this method, couples who plan to have children should undertake genetic counseling before the procedure to be informed of the risk of congenital anomalies.

Peer-review: Externally peer-reviewed.

\section{Authors Contributions}

Concept: F.G., S.G., Design: F.G., S.G., Literature search: F.G., Writing: F.G., S.G.

Conflict of Interest: No conflict of interest was declared by the authors.

Financial Disclosure: The authors declared that this study received no financial support.

\section{References}

1. Wosnitzer M, Goldstein M, Hardy MP. Review of Azoospermia. Spermatogenesis 2014;4:e28218.

2. Lehti MS, Sironen A. Formation and function of the manchette and flagellum during spermatogenesis. Reproduction 2016;151:R43-54.

3. Ramasamy R, Yagan N, Schlegel PN. Structural and functional changes to the testis after conventional versus microdissection testicular sperm extraction. Urology 2005;65:1190-1194.

4. Ogura A, Yanagimachi R. Round spermatid nuclei injected into hamster oocytes from pronuclei and participate in syngamy. Biol Reprod 1993;48:219-225.

5. Tanaka A, Nagayoshi M, Takemoto $Y$, Tanaka I, Kusunoki H, Watanabe S, Kuroda $K$, Takeda $S$, Ito $M$, Yanagimachi R. Fourteen babies born after round spermatid injection into human oocytes. Proc Natl Acad Sci U S A 2015;112:14629-14634.

6. Aire TA. Spermatogenesis and Spermiogenesis, Birds. In: Skinner MKBT-E of R (Second E, editor. Oxford: Academic Press; 2018. p. 313-320.

7. Hermo L, Oliveira RL, Smith CE, Bergeron JJM. Differential Expression of Golgi Proteins During Spermatogenesis. In: Skinner MKBT-E of R (Second E, editor. Oxford: Academic Press; 2018. p. 59-71.

8. Kızılay F, Altay B. Spermatogenesis, spermiogenesis and clinical reflections. Androl Bul 2019;21:177-184.

9. Edwards RG, Tarin JJ, Dean N, Hirsch A, Tan SL. Are spermatid injections into human oocytes now mandatory? Hum Reprod 1994;9:2217-2219. 
10. Tesarik J, Mendoza C. Spermatid injection into human oocytes. I. Laboratory techniques and special features of zygote development. Hum Reprod 1996;11:772-779.

11. Gandini $L$, Lenzi $A$, Lombardo $F$, Pacifici $R$, Dondero F. Immature germ cell separation using a modified discontinuous Percoll gradient technique in human semen. Hum Reprod 1999;14:1022-1027.

12. Liu Y, Niu M, Yao C, Hai Y, Yuan Q, Liu Y, Guo Y, Li Z, He Z. Fractionation of human spermatogenic cells using STA-PUT gravity sedimentation and their miRNA profiling. Sci Rep 2015;5:8084.

13. Miller RG, Phillips RA. Separation of cells by velocity sedimentation. J Cell Physiol 1969;73:191-201.

14. Bastos H, Lassalle B, Chicheportiche A, Riou L, Testart J, Allemand I, Fouchet P. Flow cytometric characterization of viable meiotic and postmeiotic cells by Hoechst 33342 in mouse spermatogenesis. Cytometry A 2005;65:40-49.

15. Mendoza $C$, Benkhalifa $M$, Cohen-Bacrie $P$, Hazout $A$, Ménézo $Y$, Tesarik J. Combined use of proacrosin immunocytochemistry and autosomal DNA in situ hybridisation for evaluation of human ejaculated germ cells. Zygote 1996;4:279-283.

16. Barak $Y$, Kogosowski A, Goldman $S$, Soffer $Y$, Gonen $Y$, Tesarik J. Pregnancy and birth after transfer of embryos that developed from single-nucleated zygotes obtained by injection of round spermatids into oocytes. Fertil Steril 1998;70:67-70.

17. Tanaka A, Suzuki $K$, Nagayoshi M, Tanaka A, Takemoto $Y$, Watanabe $S$, Takeda S, Irahara M, Kuji N, Yamagata Z, Yanagimachi R. Ninety babies born after round spermatid injection into oocytes: survey of their development from fertilization to 2 years of age. Fertil Steril 2018;110:443-451.

18. Aslam I, Fishel S, Green S, Campbell A, Garratt L, McDermott H, Dowell K, Thornton $S$. Can we justify spermatid microinjection for severe male factor infertility? Hum Reprod Update 1998;4:213-222.
19. Sousa M, Cremades N, Silva J, Oliveira C, Ferraz L, Teixeira da Silva J, Viana $P$, Barros A. Predictive value of testicular histology in secretory azoospermic subgroups and clinical outcome after microinjection of fresh and frozenthawed sperm and spermatids. Hum Reprod 2002;17:1800-1810.

20. Struijk RB, De Winter-Korver CM, van Daalen SKM, Hooibrink B, Repping $S$, van Pelt AMM. Simultaneous Purification of Round and Elongated Spermatids from Testis Tissue Using a FACS-Based DNA Ploidy Assay. Cytometry A 2019;95:309-313.

21. Ogonuki $N$, Inoue $H$, Matoba $S$, Kurotaki $Y K$, Kassai $H$, Abe $Y$, Sasaki $E$, Aiba A, Ogura A. Oocyte-activating capacity of fresh and frozen-thawed spermatids in the common marmoset (Callithrix jacchus). Mol Reprod Dev 2018;85:376-386.

22. Al-Hasani S, Ludwig M, Palermo I, Küpker W, Sandmann J, Johannisson R, Fornara P, Sturm R, Bals-Pratsch M, Bauer O, Diedrich K. Intracytoplasmic injection of round and elongated spermatids from azoospermic patients: results and review. Hum Reprod 1999;14 Suppl 1:97-107.

23. Oakes CC, La Salle S, Smiraglia DJ, Robaire B, Trasler JM. Developmental acquisition of genome-wide DNA methylation occurs prior to meiosis in male germ cells. Dev Biol 2007;307:368-379.

24. Shamanski FL, Kimura Y, Lavoir MC, Pedersen RA, Yanagimachi R. Status of genomic imprinting in mouse spermatids. Hum Reprod 1999;14:1050-1056.

25. Bonduelle M, Liebaers I, Deketelaere V, Derde MP, Camus M, Devroey P, Van Steirteghem A. Neonatal data on a cohort of 2889 infants born after ICSI (1991-1999) and of 2995 infants born after IVF (1983-1999). Hum Reprod 2002;17:671-694.

26. Ludwig M, Diedrich K. Follow-up of children born after assisted reproductive technologies. Reprod Biomed Online 2002;5:317-322. 